\title{
Turnover rate of tear-film lipid layer determined by fluorophotometry
}

\author{
H Mochizuki, ${ }^{1}$ M Yamada, ${ }^{1}$ S Hatou, ${ }^{1,2}$ K Tsubota ${ }^{2}$
}

${ }^{1}$ Division for Vision Research, National Institute of Sensory Organs, National Tokyo Medical Center, Tokyo, Japan;

${ }^{2}$ Department of Ophthalmology, Keio University School of Medicine, Tokyo, Japan

Correspondence to: Dr M Yamada, Division for Vision Research, National Institute of Sensory Organs, National Tokyo Medical Center, 2-5-1

Higashigaoka, Meguro-ku, Tokyo 152-8902, Japan;

yamadamasakazu@kankakuki. go.jp

Accepted 1 July 2009

Published Online First

18 August 2009

\section{ABSTRACT}

Aim: This study was performed to independently assess the turnover rates of aqueous and lipid layers of the tear film.

Methods: Two fluorescent dyes, fluorescein sodium and 5-dodecanoylaminofluorescein (DAF), which is a freefatty-acid conjugate of fluorescein, were applied to the right eye of 12 healthy volunteers. Fluorescent intensity of the precorneal tear film was measured at the central cornea every minute for 10 min for fluorescein sodium, and every $5 \mathrm{~min}$ for $50 \mathrm{~min}$ for DAF. The turnover rate was calculated by plotting fluorescent intensity against time in a semilog plot and expressed as \%/min.

Results: Turnover rates of fluorescein sodium and DAF were 10.3 (SD 3.7)\%/min and $0.93(0.36) \% / \mathrm{min}$, respectively. The turnover rate of DAF was significantly lower than that of fluorescein sodium $(p<0.05$, MannWhitney test). The turnover rate of DAF positively correlated with that of fluorescein sodium $(r=0.93$, $\mathrm{p}<0.05$ ).

Conclusion: Our results indicate that the turnover of lipids in tears is much slower than the aqueous flow of tears, and that this lipid turnover is associated with the aqueous flow of tears in healthy adults.

The precorneal tear film has traditionally been described as consisting of an outer lipid layer, a middle aqueous layer and an inner mucus layer. Although this remains valid, some modifications have been proposed. ${ }^{1-3}$ In the current model of the tear film, the aqueous-mucin layer is covered by two thin layers of lipids. Polar lipids such as phospholipids lie adjacent to the aqueous-mucin layer, and non-polar lipids such as cholesterol and wax ester are present at the tear-air interface. In addition, tears contain proteins that possess lipidbinding properties, such as tear lipocalin. ${ }^{4-6}$ Although lipids in tears are primarily located in the tear-film lipid layer, some lipids are presumably bound by lipocalin in the aqueous layer. Tear lipocalin is thought to have an important role in stabilising the tear-film lipid layer by transferring lipids to it from the aqueous layer. ${ }^{4-6}$

Despite comprising a very small proportion of the overall tear-film thickness, the lipid layer is important for retarding evaporation and maintaining tear-film stability. ${ }^{2}{ }^{3}$ Where the lipid layer is absent or where the integrity of the lipid layer is compromised, the evaporation rate of tears increases, accompanied by tear-film instability. ${ }^{78}$ To assess the lipid layer of tears, several techniques have been developed, including observation of lipid layer characteristics by interferometric methods, ${ }^{9-11}$ quantitative measurement of meibomian lipid on the lid margin by meibometry ${ }^{12}{ }^{13}$ and measurement of evaporation from the ocular surface..$^{14-16}$ Of these, observation of lipid layer characteristics by interferometric methods has been well established. ${ }^{9-11} 17$ In various pathological conditions, such as meibomian gland dysfunction, the appearance of the lipid layer can change. Lipid layer thickness, measured by interferometry, has been reported to correlate with tear-film evaporation, tear-film breakup time, and clinical symptoms. ${ }^{818}$ We have previously reported that the concentration of lipocalin in tears from patients with meibomian gland dysfunction was significantly lower than in normal controls. ${ }^{19}$ Thus, lipids in tears, both in the lipid layer and in the aqueous layer held by lipocalin, are important when considering the pathophysiology of evaporative dry eye, such as meibomian gland dysfunction. Until now, however, there has been no information about the flow rate of tear-film lipid layer.

Aqueous tear flow is determined by several aspects of tear dynamics including tear production, tear volume, tear evaporation and tear outflow. ${ }^{20}$ Tear flow can be assessed by introducing a dye or radioactive substance into the conjunctival sac and measuring the decay in concentration over a certain period. Since the report of Mishima et $a l^{21}$ fluorophotometric measurement using fluorescein sodium as a tracer has been the gold standard to quantify tear flow. ${ }^{16}{ }^{20}$ The elimination rate of fluorescein sodium essentially represents the bulk aqueous flow because the dye is hydrophilic; however, the turnover of a certain tear component may not parallel the bulk aqueous flow. For example, we recently reported differences between the bulk aqueous flow of hyaluronic acid and the turnover of hyaluronic acid, suggesting that hyaluronic acid remains on the ocular surface independent of the bulk aqueous flow. ${ }^{22}$ Accordingly, we hypothesised that the flow rate of the tear lipid layer might be different from that of aqueous tear layer.

In this study, we tested this hypothesis using fluorescein sodium and a free-fatty-acid conjugate of fluorescein. Fluorescein was used to assess the aqueous flow, and the conjugated dye was used as a tracer to determine the flow rate of the tear lipid layer.

\section{METHODS}

\section{Fluorescent dye and fluorophotometer}

5-Dodecanoylaminofluorescein (DAF; Molecular Probes, Eugene, Oregon) is a lipophilic and waterinsoluble free-fatty-acid conjugate of fluorescein. This dye has the longest-wavelength absorption maximum at $495 \mathrm{~nm}$, and an emission spectrum that peaks at $518 \mathrm{~nm}$. A DAF emulsion $(50 \mathrm{mg} / \mathrm{ml})$ was prepared in sterile $0.067 \mathrm{M}$ phosphate-buffered saline (PBS), $\mathrm{pH}$ 7.4, with 1\% Tween 80 
(Sigma-Aldrich, St Louis, Missouri). Fluorescein sodium (SigmaAldrich) was dissolved in sterile $0.067 \mathrm{M} \mathrm{PBS}$ and used as a tracer of the tear aqueous layer. No signs of inflammation or damage were detected either immediately or after $24 \mathrm{~h}$ by instilling five drops of $5 \%$ DAF emulsion at $10 \mathrm{~min}$ intervals into four rabbits' eyes. Instillation of one drop of $5 \% \mathrm{DAF}$ emulsion into the eyes of four subjects caused no discomfort, and no staining or adverse effects were detected by a slit-lamp examination. To test the effect of DAF emulsion on tear-film stability, we measured tear-film break-up time (BUT) after instilling a $1 \mu \mathrm{l}$ of DAF emulsion $(50 \mathrm{mg} / \mathrm{ml})$. The DAF-BUT was $21.6(10.4) \mathrm{s}(\mathrm{n}=20)$, which was longer than fluorescein BUT (13.1 (3.6) s) measured by instilling $1 \mu \mathrm{l}$ of fluorescein sodium solution $(50 \mu \mathrm{g} / \mathrm{ml})$ on a different day. From these preliminary experiments, a DAF emulsion was considered to have no apparent adverse effects on the ocular surface or the tear film.

A modified Bligh and Dyer procedure was performed to test the nature of these dyes. Ten microlitres of $5 \%$ DAF emulsion and $0.5 \%$ fluorescein sodium solution were placed in a test tube with $1 \mathrm{ml}$ of a 2:1 chloroform:methanol solvent (Wako, Osaka, Japan). After adding $0.4 \mathrm{ml}$ of water, the tubes were vortexed for $30 \mathrm{~s}$. The solvent formed two layers-an upper aqueous layer and a lower lipid layer. As expected, the fluorescent yellowish colour of DAF was seen in the lower lipid layer, whereas the dye colour of fluorescein sodium was seen in the upper aqueous layer (fig 1). Spectrophotometric measurements $(495 \mathrm{~nm})$ revealed that $99 \%$ of DAF was located in the lower lipid layer, and $97 \%$ of fluorescein sodium was in the upper aqueous layer.

A commercial slit-lamp fluorophotometer (Anterior Fluorometer FL-500, Kowa, Tokyo) was used to quantify fluorescence intensity. The illuminating light was focused as a $2 \mathrm{~mm}$ diameter circle on the surface of the cornea. The emitted light passed through a band interference filter centred on $565 \mathrm{~nm}$ (half bandwidth $25 \mathrm{~nm}$ ) and was directed to a photomultiplier tube with the band interference filter centred on a wavelength of $490 \mathrm{~nm}$ (half bandwidth $30 \mathrm{~nm}$ ).

DAF emulsion $(50 \mathrm{mg} / \mathrm{ml}$ ) was diluted in PBS to produce standards ranging from 0.01 to $50 \mathrm{mg} / \mathrm{ml}$ for calibration. Fluorescein sodium solution $(5 \mathrm{mg} / \mathrm{ml})$ was diluted to produce standards ranging from 0.1 to $50 \mu \mathrm{g} / \mathrm{ml}$ in the same fashion. A cuvette was constructed by gluing together two microscopic slides and two cover glasses. The cover glasses were sandwiched by the two slides to provide a $12-15 \mu \mathrm{m}$ thick space for the fluid
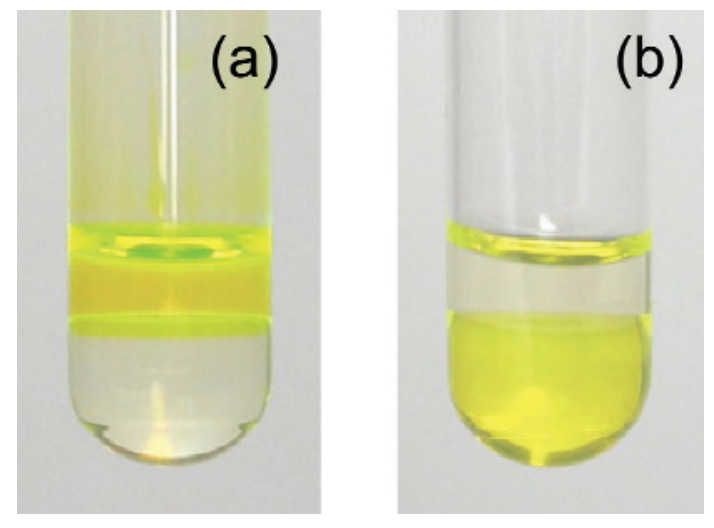

Figure 1 Fluorescein sodium (A) and 5-dodecanoylaminofluorescein (DAF) (B) after a modified Bligh and Dyer procedure. The fluorescent yellowish colour of fluorescein sodium was seen in the upper aqueous layer, whereas the dye colour of DAF was seen in the lower lipid layer. layer. A fresh cuvette was used for each solution. The standards $(10 \mu \mathrm{l})$ were added to the cuvettes, and fluorescence intensity was measured by the slit-lamp fluorophotometer. The interaction of DAF or fluorescein sodium with proteins was also tested by diluting the standards in PBS containing 10\% fetal bovine serum (FBS).

\section{Measurement of turnover rate}

Twelve healthy volunteers (six males and six females) aged 21 to 47 years (mean 32.6 (SD 8.2) years), who had no history of eye disease except for refractive errors, were enrolled in the study. One of the authors $(\mathrm{SH})$ performed a routine ocular examination on all subjects, followed by an examination of the ocular surface, including Schirmer testing and measurement of tear-film break-up time (BUT). For vital staining, $2 \mu$ l of a saline solution containing $1 \%$ fluorescein was used. All subjects had more than $5 \mathrm{~mm}$ of Schirmer strip wetting, more than $5 \mathrm{~s}$ in tear-film BUT, and no apparent fluorescein staining of cornea and conjunctiva. The Marx lines, which run along the eyelid margin determined by fluorescein staining, were normal in all subjects. ${ }^{23}$ The guidelines of the World Medical Association Declaration of Helsinki were followed. The subjects received a full explanation of the procedures and provided their informed consent for participation prior to the experiment. The protocol was approved by the institutional review board of National Tokyo Medical Center (R-07-011: Assessment of layer-by-layer tear dynamics by fluorophotometry), and all subjects provided written informed consent.

The subjects were seated in front of the fluorophotometer. The instrument was focused on the central cornea, and background fluorescence intensity was measured. A volume of $1 \mu \mathrm{l}$ of DAF emulsion $(50 \mathrm{mg} / \mathrm{ml})$ or fluorescein sodium solution $(50 \mu \mathrm{g} / \mathrm{ml})$ was applied to the right eye using an Eppendorf micropipette without making contact with the ocular surface. The subjects were instructed to blink several times to ensure mixing of the dye. Fluorescence intensity of the precorneal tear film was measured at the central cornea. When fluorescein sodium solution was instilled into the eye, fluorescence intensity was measured every minute for $10 \mathrm{~min}$. When DAF emulsion was instilled, measurements were repeated every $5 \mathrm{~min}$ for $50 \mathrm{~min}$ because of a slower decay of intensity. Measurements of fluorescein sodium and DAF were done on different days. Measurements of DAF were repeated on three different days in three subjects to evaluate the repeatability of the test.

The turnover rate was determined by plotting fluorescence intensity against time in a semilog plot, $\mathrm{F}=\mathrm{F}_{0} \exp (-\mathrm{kt})$, where $\mathrm{F}=$ fluorescence intensity at time $(\mathrm{t}), \mathrm{F}_{0}$ fluorescence intensity at time zero, $\mathrm{k}=$ turnover rate and $\mathrm{t}=$ time $(\mathrm{min})$. The turnover rate was calculated for all tests and expressed as $\% / \mathrm{min}$. The regression fit of the log of fluorescence intensity was recorded as the regression coefficient.

When DAF values were plotted, the regression line was straight. On the other hand, when fluorescein sodium values were plotted, some regression lines were biphasic, representing an initial faster turnover rate and a subsequent slower turnover rate. In cases in which the turnover rate of fluorescein sodium became biphasic, the subsequent slower turnover rate was used as the flow rate.

All results are presented as the mean (SD). Statistical significance was calculated by comparing results using the Mann-Whitney test. A $p$ value of $<0.05$ was considered statistically significant. 


\section{RESULTS}

\section{Calibration of DAF and fluorescein sodium}

The relationship between fluorescence intensity and DAF concentration was linear (fig $2, \mathrm{r}^{2}=0.991$ ). The data generated by this method were consistent and reproducible. Fluorescence intensity was unaffected by the presence of 10\% FBS (data not shown). Similar results were obtained when the fluorescein sodium standards were tested (data not shown).

\section{Turnover rate}

A representative result of turnover rate obtained from one subject is shown in fig 3 . Fluorescence intensity of fluorescein sodium decayed with time at a flow rate of $14.5 \% / \mathrm{min}$. In the presented case, the fluorescence decay rate of DAF was much lower $(1.14 \% / \mathrm{min})$ that that of fluorescein sodium. To test the reproducibility of the DAF method, the measurement was repeated three times in three eyes of three subjects. The coefficient of variance of the measurements was lower than 0.1 in all cases (mean $=0.07, \mathrm{n}=3$ ), which ensured the reproducibility of the measurement.

The turnover rates of DAF and fluorescein sodium were 0.93 $(0.36) \% / \mathrm{min}$ and $10.3(3.7) \% / \mathrm{min}$, respectively (table 1$)$. The turnover rate of DAF was significantly lower than that of fluorescein sodium $(\mathrm{p}<0.05)$.

The turnover rate of DAF positively correlated with that of fluorescein sodium (fig 4; $\mathrm{r}^{2}=0.87, \mathrm{p}<0.05$ ).

\section{DISCUSSION}

We used fluorescein sodium and DAF to assess independently the turnover rates of aqueous and lipid layers of the tear film. To the best of our knowledge, this is the first study to address the turnover rate of the tear-film lipid layer.

There are potential limitations when interpreting the results of the present study, of which one is that DAF is a free-fattyacid conjugate of fluorescein. Tear lipids are known to consist of various classes of lipids, such as wax esters, cholesterol, cholesterol esters, phospholipids, glycolipids and free fatty acids. $^{2}{ }^{24}$ Because different classes of lipids have different biophysical properties, including molecular weight, hydropho-

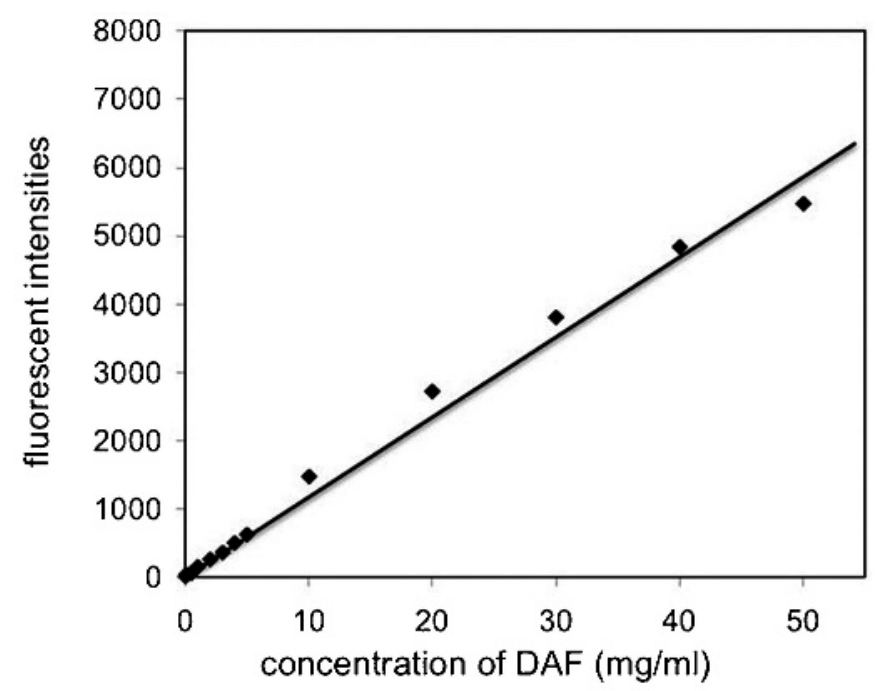

Figure 2 Plot of fluorescence intensity and 5-

dodecanoylaminofluorescein (DAF) concentration. The relationship between fluorescence intensity and DAF concentration was linear $\left(r^{2}=0.991\right)$.

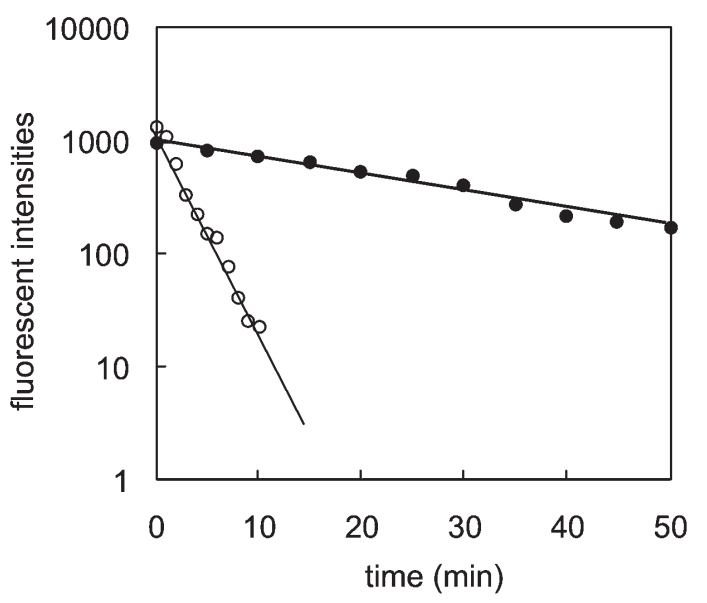

Figure 3 Representative result of turnover rate obtained from one subject. Fluorescence intensity of 5-dodecanoylaminofluorescein (black circle) decayed with time at a flow rate of $1.14 \% / \mathrm{min}$, which was lower than that of fluorescein sodium (14.5\%/min; white circle).

bicity, viscosity and binding capacity to tear lipocalin, ${ }^{24} 25$ the turnover of all lipids in tears may not be the same as that of DAF. Another limitation is the usage of a $5 \%$ DAF emulsion containing Tween 80. Although the addition of a surfactant was essential to make the emulsion of DAF, we considered it undesirable because it might disrupt the tear-film stability. We minimised the amount of Tween 80 in the emulsion, and the applied volume of DAF emulsion into the eye, to avoid the effect of a surfactant as much as possible.

The most interesting finding was that the turnover rate of DAF $(0.9(0.4) \% / \mathrm{min})$ was approximately $9 \%$ that of fluorescein sodium $(10.3(3.8) \% / \mathrm{min})$, indicating that the turnover of lipids in tears is much slower than the aqueous flow of tears. The discrepancy between the results of the dyes may be explained by the small bulk of the tear-film lipid layer compared with that of the marginal reservoirs. ${ }^{2}$ Using meibometry, Chew et a ${ }^{12} 13$ estimated that approximately $300 \mu \mathrm{g}$ of lipid is present in the marginal reservoir and calculated that the preocular tear film contains approximately $9 \mu \mathrm{g}$ of lipids. It has been estimated that the volume of the preocular tear film is $1-1.5 \mu \mathrm{l}$ and that the total volume of tear fluid is $7-10 \mu 1 .{ }^{20}$ Our results confirm

Table 1 Turnover rates of a topically applied $(1 \mu \mathrm{l}) 50 \mathrm{mg} / \mathrm{ml} 5$ dodecanoylaminofluorescein (DAF) emulsion or $50 \mu \mathrm{g} / \mathrm{ml}$ fluorescein sodium solution observed in 12 subjects

\begin{tabular}{lll}
\hline \multirow{2}{*}{ Subject no } & \multicolumn{2}{l}{ Turnover rate $(\% /$ min) } \\
\cline { 2 - 3 } & DAF & Fluorescein sodium \\
\hline 1 & 0.51 & 5.2 \\
2 & 0.46 & 4.4 \\
3 & 0.54 & 7.1 \\
4 & 0.61 & 7.2 \\
5 & 0.84 & 9.7 \\
6 & 0.93 & 10.0 \\
7 & 0.84 & 10.2 \\
8 & 1.12 & 11.5 \\
9 & 1.60 & 14.7 \\
10 & 1.31 & 12.9 \\
11 & 1.24 & 15.8 \\
12 & 1.14 & 14.5 \\
Mean (SD) & $0.93(0.36)$ & $10.3(3.7)$
\end{tabular}

The turnover rate of DAF was significantly lower than that of fluorescein sodium $(p<0.05)$. 


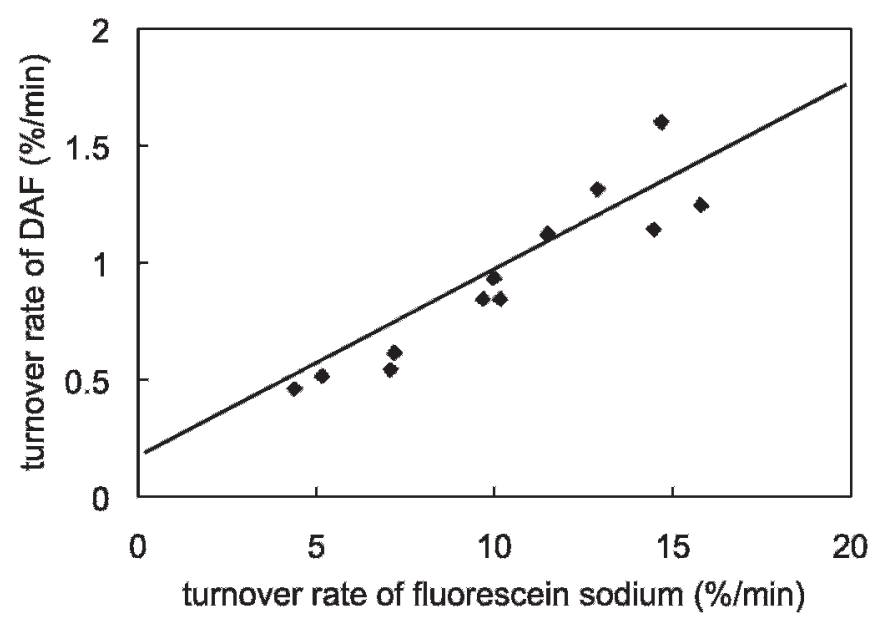

Figure 4 Relationship between the turnover rates of 5 dodecanoylaminofluorescein (DAF) and fluorescein sodium. The DAF turnover rate positively correlated with that of fluorescein sodium $\left(r^{2}=0.87, p<0.05\right)$.

that the lipid layer, despite comprising a very small proportion of the overall tear film, is a distinct component of the tear film from others.

Another important finding of our study is that the turnover rate of DAF correlates well with that of fluorescein sodium. This result indicates that subjects with a high turnover rate of aqueous tears tend to have a high turnover rate of lipids in tears. Until now, the mechanism for excretion of tear lipids has not been fully understood. Bron et $a l^{2}$ stated that excretion most likely occurs by bulk flow over the lid margin and onto the neighbouring lid skin and lashes. They also indicated that lipids may be excreted by diffusion from the tear-film lipid layer into the aqueous phase of the tear film. In the latter, the biochemical interaction between proteins and lipids may have a role in transferring and scavenging lipids in tears. ${ }^{24-6}$ Our results suggest that lipid turnover in tears is, at least partially, associated with the aqueous flow of tears. The association between them may be due to the facilitated excretion onto the lid skin, because a higher aqueous flow of tears is sometimes associated with a larger tear volume. ${ }^{16}$ Alternatively, the association may reflect the turnover of a lipid fraction bound by lipocalin in the aqueous layer. ${ }^{4-6}$ Our current methodology, however, is not able to distinguish two routes of lipid excretion from tears.

The data presented in the current study were obtained from healthy adults. Lipid turnover in tears may be different in older subjects or in those with meibomian gland dysfunction. The anterior displacements of muco-cutaneous junction are associated with ageing and the presence of meibomian gland dysfunction. ${ }^{23}$ In this situation, some meibomian gland orifices are open posterior to the muco-cutaneous junction. Therefore, the dynamics of tear lipids excretions in these cases may differ from normal subjects. Further investigations have been planned to clarify these issues.

Funding: This study was supported in part by a grant from the Ministry of Health, Labour and Welfare, Japan, and by a grant from the Ministry of Education, Culture, Sports, Science, and Technology, Japan.

Competing interests: None.

Ethics approval: Ethics approval was provided by National Tokyo Medical Center.

Patient consent: Obtained.

Provenance and peer review: Not commissioned; externally peer reviewed.

\section{REFERENCES}

1. Wolff $\mathbf{E}$. The muco-cutaneous junction of the lid margin and the distribution of the tear fluid. Trans Ophthalmol Soc UK 1946;66:291-308.

2. Bron AJ, Tiffany JM, Gouveia SM, et al. Functional aspects of the tear film lipid layer. Exp Eye Res 2004;78:347-60.

3. McCulley JP, Shine W. A compositional based model for the tear film lipid layer. Trans Am Ophthalmol Soc 1997:95:79-88.

4. Glasgow BJ, Marshall G, Gasymov OK, et al. Tear lipocalins: potential lipid scavengers for the corneal surface. Invest Ophthalmol Vis Sci 1990;40:3100-7.

5. Glasgow BJ, Abduragimov AR, Farahbakhsh ZT, et al. Tear lipocalin bind a broad array of lipid ligands. Tear lipocalins bind a broad array of lipid ligands. Curr Eye Res 1995; 14:363-72.

6. Millar TJ, Mudgil P, Butovich IA, et al. Adsorption of human tear lipocalin to human meibomian lipid films. Invest Ophthalmol Vis Sci 2009;50:140-51.

7. Mishima S, Maurice DM. The oily layer of the tear film and evaporation from the corneal surface. Exp Eye Res 1961;1:39-45.

8. Craig JP, Tomlinson A. Importance of the lipid layer in human tear film stability and evaporation. Optom Vis Sci 1997;74:8-13.

9. Norn MS. Semiquantitative interference stuffy of fatty layer of precorneal film. Acta Ophthalmol 1979;57:766-74.

10. Korb DR, Baron DF, Herman JP, et al. Tear film lipid layer thickness as a function of blinking. Cornea 1994;13:354-9.

11. Yokoi N, Takehisa Y, Kinoshita S. Correlation of tear lipid layer interference patterns with the diagnosis and severity of dry eye. Am J Ophthalmol 1996;122:818-24.

12. Chew CK, Janweiier C, Tiffany JM, et al. An instrument for quantifying meibomian lipid on the lid margin: the meibometer. Curr Eye Res 1993;12:247-54.

13. Chew CK, Hykin PG, Janweiier $C$, et al. The casual level of meibomian lipids in humans. Curr Eye Res 1993;12:255-9.

14. Rolando M, Refojo MF. Tear evaporiometer for measuring water evaporation from the tear film under controlled conditions in humans. Exp Eye Res 1983:36:25-33.

15. Tsubota K, Yamada M. Tear evaporation from the ocular surface. Invest Ophthalmol Vis Sci 1992;33:2942-50.

16. Mathers WD, Thomas ED. Tear flow and evaporation in patients with and without dry eye. Ophthalmology 1996;103:664-9.

17. Yokoi N, Komuro A. Non-invasive methods of assessing the tear film. Exp Eye Res 2004;78:399-407.

18. Nichols JJ, Nichols KK, Puent B, et al. Evaluation for tear film interference patterns and measures of tear break-up time. Optom Vis Sci 2002;79:363-9.

19. Yamada M, Mochizuki H, Kawai M, et al. Decreased tear lipocalin concentration in patients with meibomian gland dysfunction. Br J Ophthalmol 2005;89:803-5.

20. Lemp MA. Basic principles and classification of dry eye disorders. In: Lemp MA, Marquardt, eds. The dry eye. Berlin: Springer, 1992:101-31.

21. Mishima S, Gasset A, Klyce SD Jr, et al. Determination of tear volume and tear flow. Invest Ophthalmol Vis Sci 1966;5:264-76.

22. Mochizuki H, Yamada M, Hatou S, et al. Fluorophotometric measurement of the precorneal residence time of topically applied hyaluronic acid. $\mathrm{Br} \mathrm{J}$ Ophthalmol 2008;92:108-11.

23. Yamaguchi $\mathbf{M}$, Kutsuna $M$, Uno $T$, et al. Marx line: fluorescein staining line on the inner lid as indicator of meibomian gland dysfunction. Am J Ophthalmol 2006;141:669-75.

24. McCulley JP, Shine WE. Meibomian gland and tear film lipids: structure, function and control. Adv Exp Med Biol 2002;506:373-8.

25. Nagyová B, Tiffany JM. Components responsible for the surface tension of human tears. Curr Eye Res 1999;19:4-11. 